\title{
hidroeléctrica de Chivor
} Colombia

GABRIEL TOBAR GUZMAN, AICEMCO

$531-72$

INTRODUCCION

Colombia, país de grandes recursos hidráulicos, se ha venido preocupando permanentemente del aprovechamiento de dichos recursos.

Con este fin, las principales empresas de energía del país vieron la necesidad de unir sus fuerzas y formar así la sociedad "Interconexión Eléctrica, S. A.» (ISA).

\section{sinopsis}

Con el proyecto de Chivor comenzó la construcción de la Central Hidroeléctrica más gransituada a $160 \mathrm{~km}$ al noroeste de Bogotá, sobre el río Batá, en el Departamento de Boyacá, cerca de Santa María, tendrá una capacidad de 1.000 .000 de kilovatios y su desarrolio se hará en dos etapas de $500.000 \mathrm{~kW}$ de capacidad cada una. La primera unidad de $125.000 \mathrm{~kW}$ en tró en servicio a mediados de 1976 y la cuarta unidad, para completar la primera etapa de $500.000 \mathrm{~kW}$, estaba lista en diciembre de 1976. La construcción de las unidades 5 a 8 están programadas para entrar en servicio en 1978 .

En este artículo se describen: la presa La Esmeralda, de enrocado con núcleo impermeable de arcilla, y $237 \mathrm{~m}$ de altura, considerada la tercera más alta del mundo de este tipo; el embalse; el rebosadero; el túne de carga; la tubería de carga y la casa de máquinas.

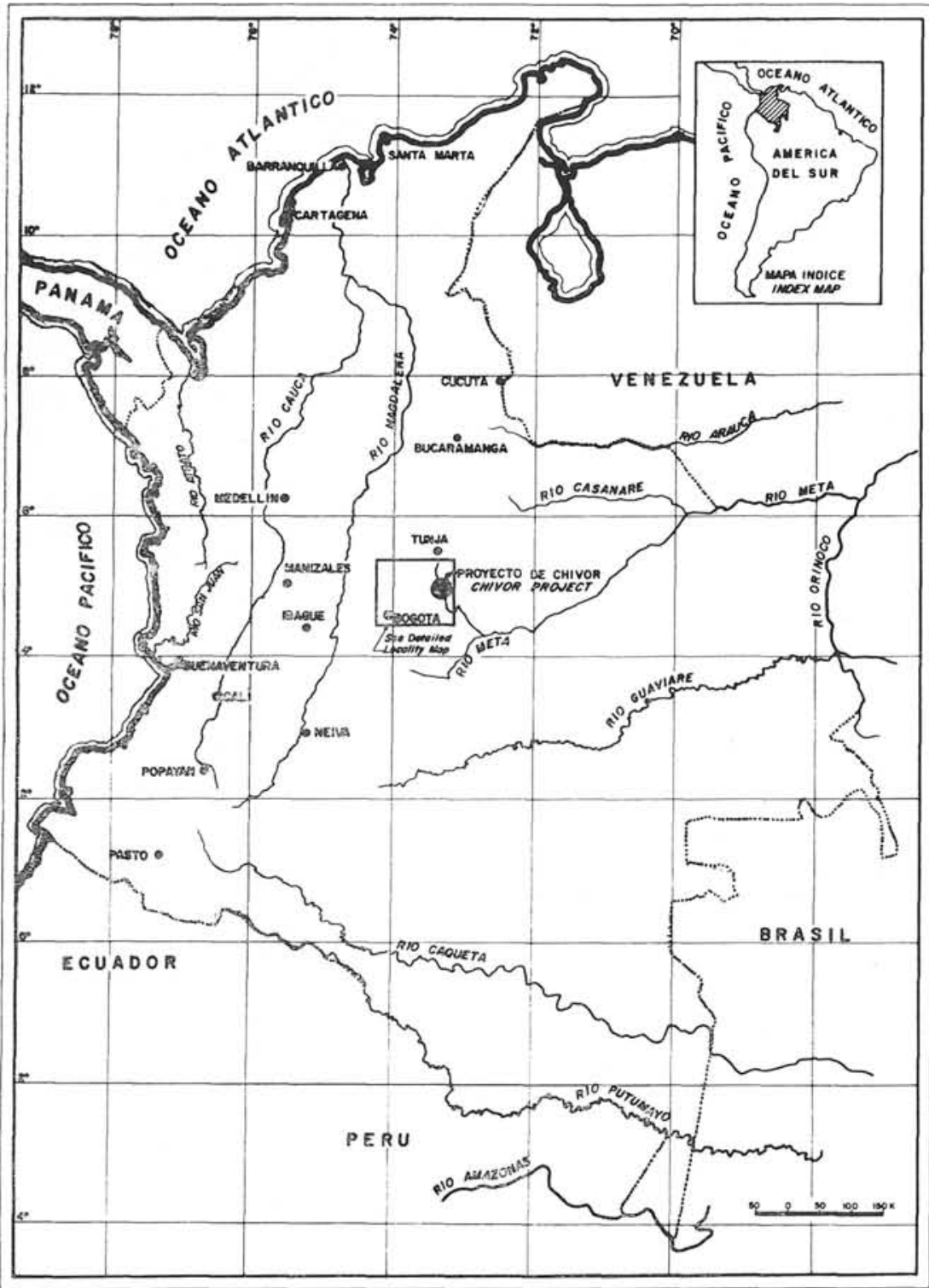

MAPA DE LA REPUBLICA DE COLOMBIA REPUBLIC OF COLOMBIA MAP 
Con el proyecto de CHIVOR, ISA, comenzó la construcción de la central hidroeléctrica más grande de Colombia. Esta central, que tendrá una capacidad de un millón de kW, se está construyendo bajo la responsabilidad de la Empresa de Energía Eléctrica de Bogotá, miembro de la sociedad ISA, y que para este importante proyecto cuenta con la colaboración de la firma de ingenieros consultores Ingetec Ltda. La construcción se desarrollará

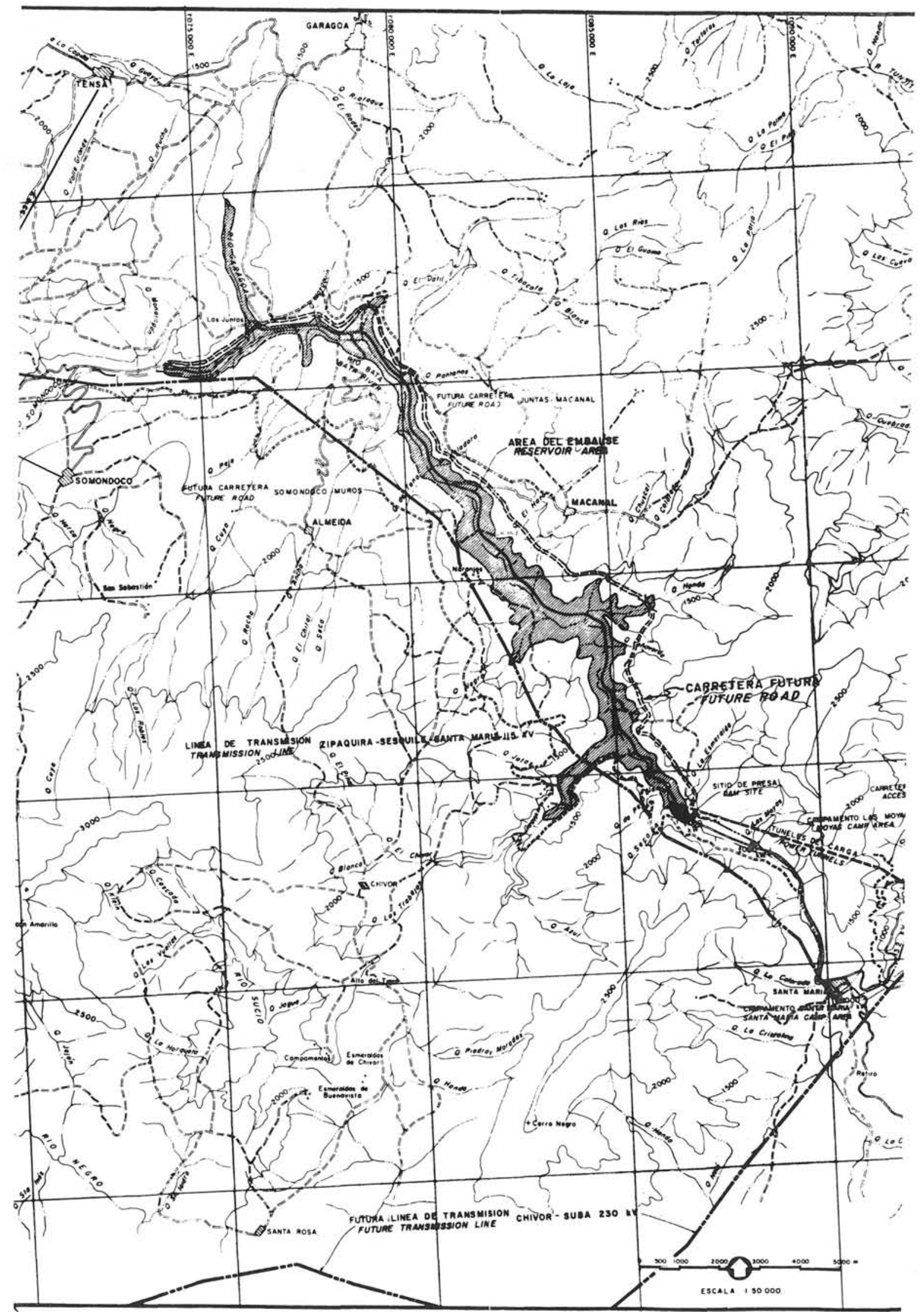




\section{DATOS GENERALES DE LA OBRA}

\section{Localización}

El proyecto de la central hidroeléctrica de Chivor está localizado a $160 \mathrm{~km}$ de Bogotá, sobre el rio Batá, en el Departamento de Boyacá, cerca de la población de Santa María, donde se encuentran entablecidas las instalaciones de los contratistas y de la interventoría. La zona fue escogida después de realizar en la misma numerosas investigaciones geoló-
gicas y estudios hidrológicos.

\section{Descripción general}

Las obras consisten, básicamente, en una presa de enrocado de $237 \mathrm{~m}$ de altura, medidos desde el nivel de cimentación. Esta presa tendrá las aguas del río Batá para permitir que, mediante $5.800 \mathrm{~m}$ de tancl y $2.100 \mathrm{~m}$ de tuberia, se puedan conducir estas aguas hasta la casa de máquinas, situada en la margen derecha del rio Lengupá, aguas arriba de San Luis de Gaceno; de esta manera se aprovecha una caída neta promedia de $756 \mathrm{~m}$.

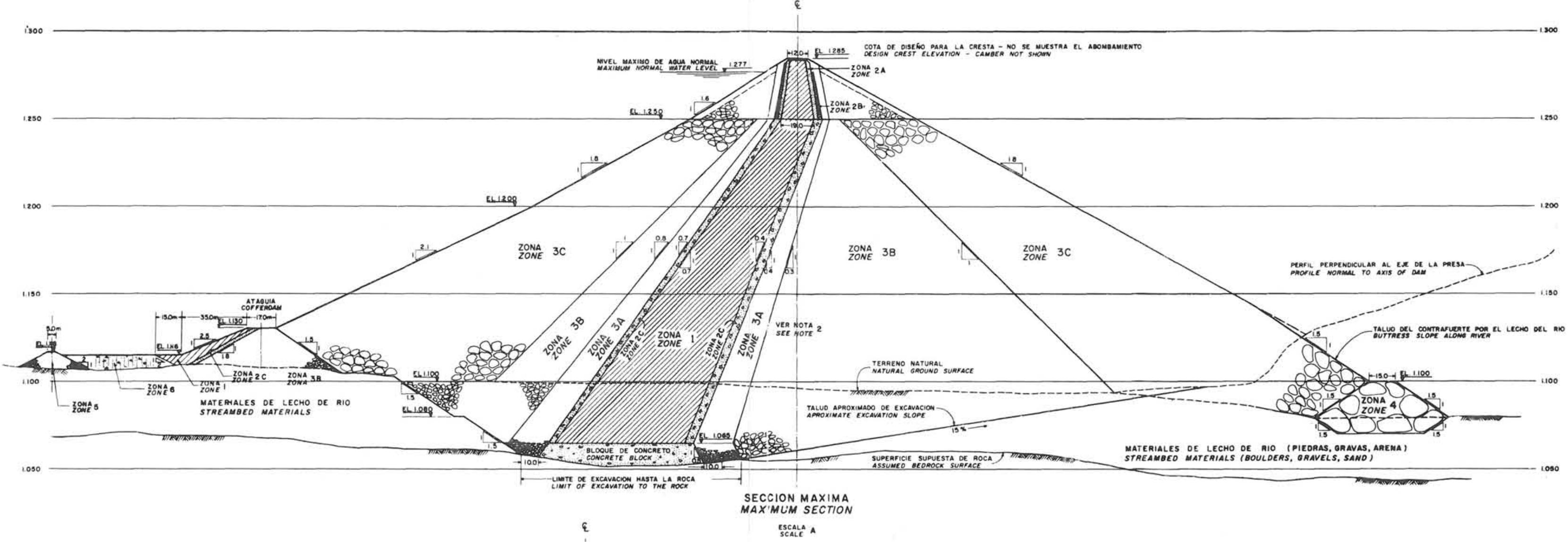

presa de La Esmeralda Escata A
Scale A

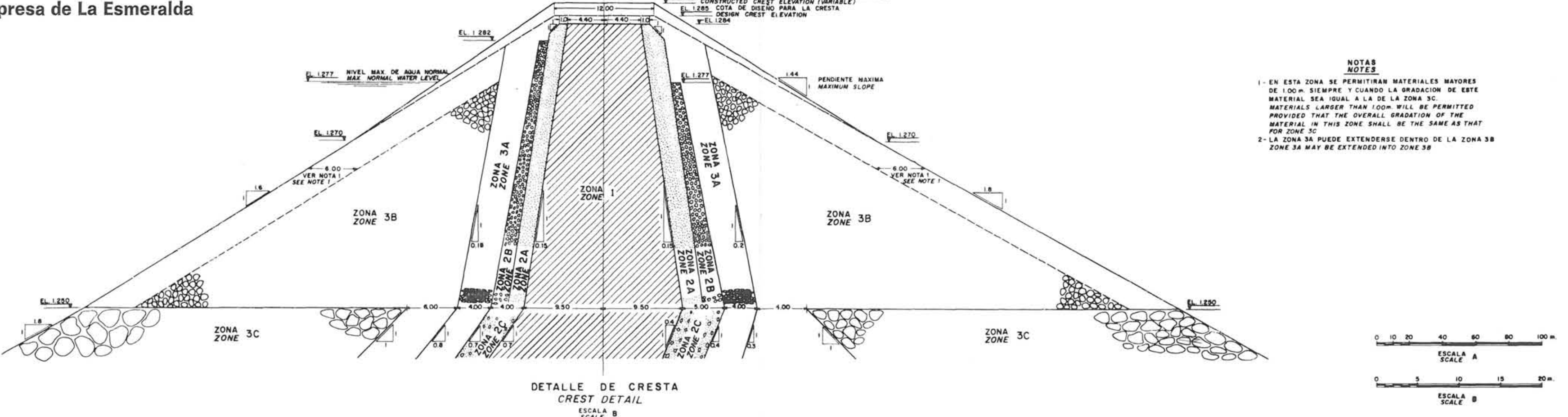


en dos etapas de $500.000 \mathrm{~kW}$ de capacidad cada una: la primera etapa entró en servicio en diciembre de 1976, y la segunda está programada para entrar en servicio en 1978.

La financiación para la construcción de la obra está asegurada con empréstitos concedidos por el Banco Internacional de Reconstrucción y Fomento (BIRF) en una cuantía de
US S 52.300.000, y por el Banco Interamericano de Desarrollo (BID), en otra cuantía de S 70.600 .000 , para así sumar un total de US \$ 210.000.000.

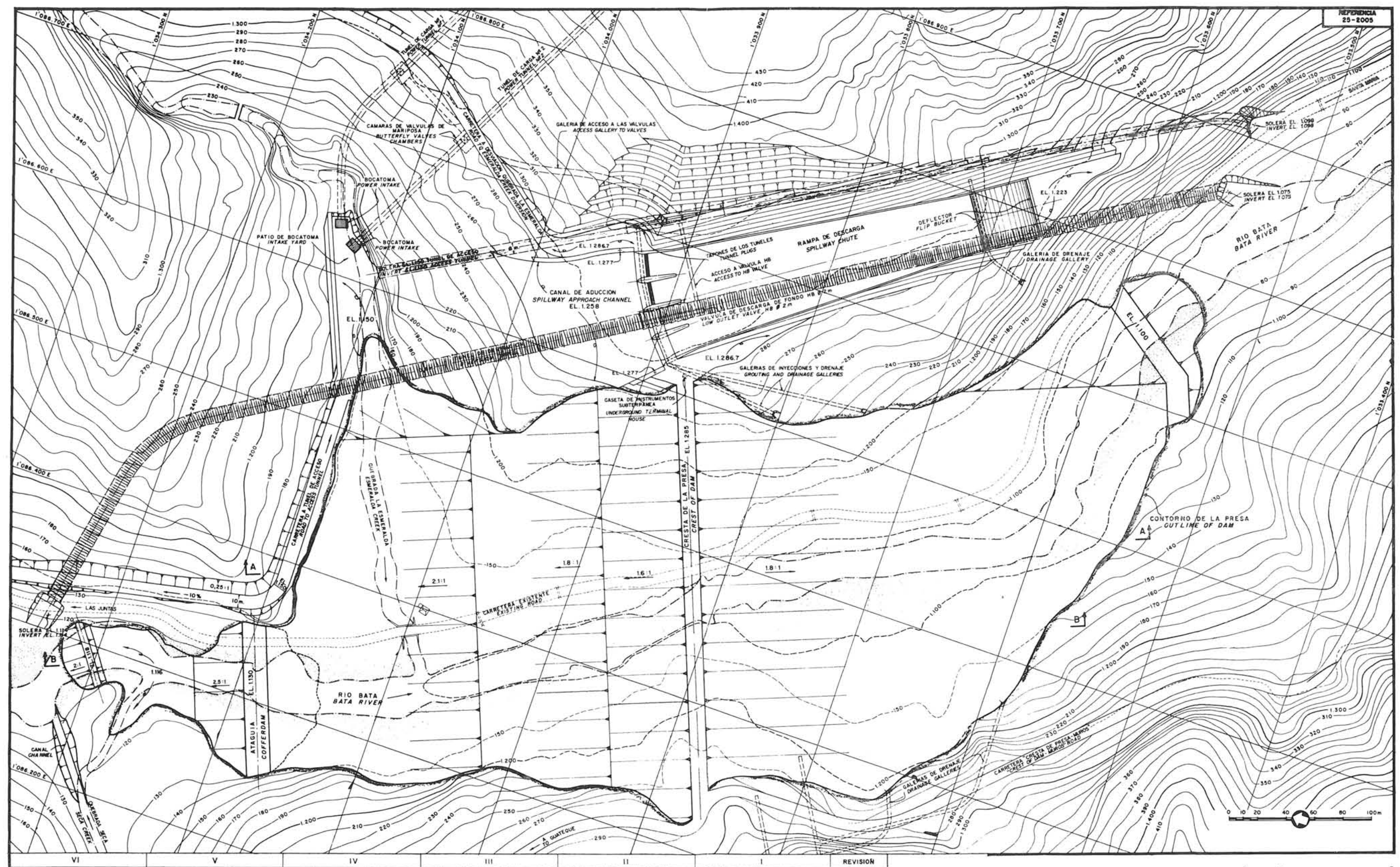

planta general 


\section{túnel y tubería de carga}
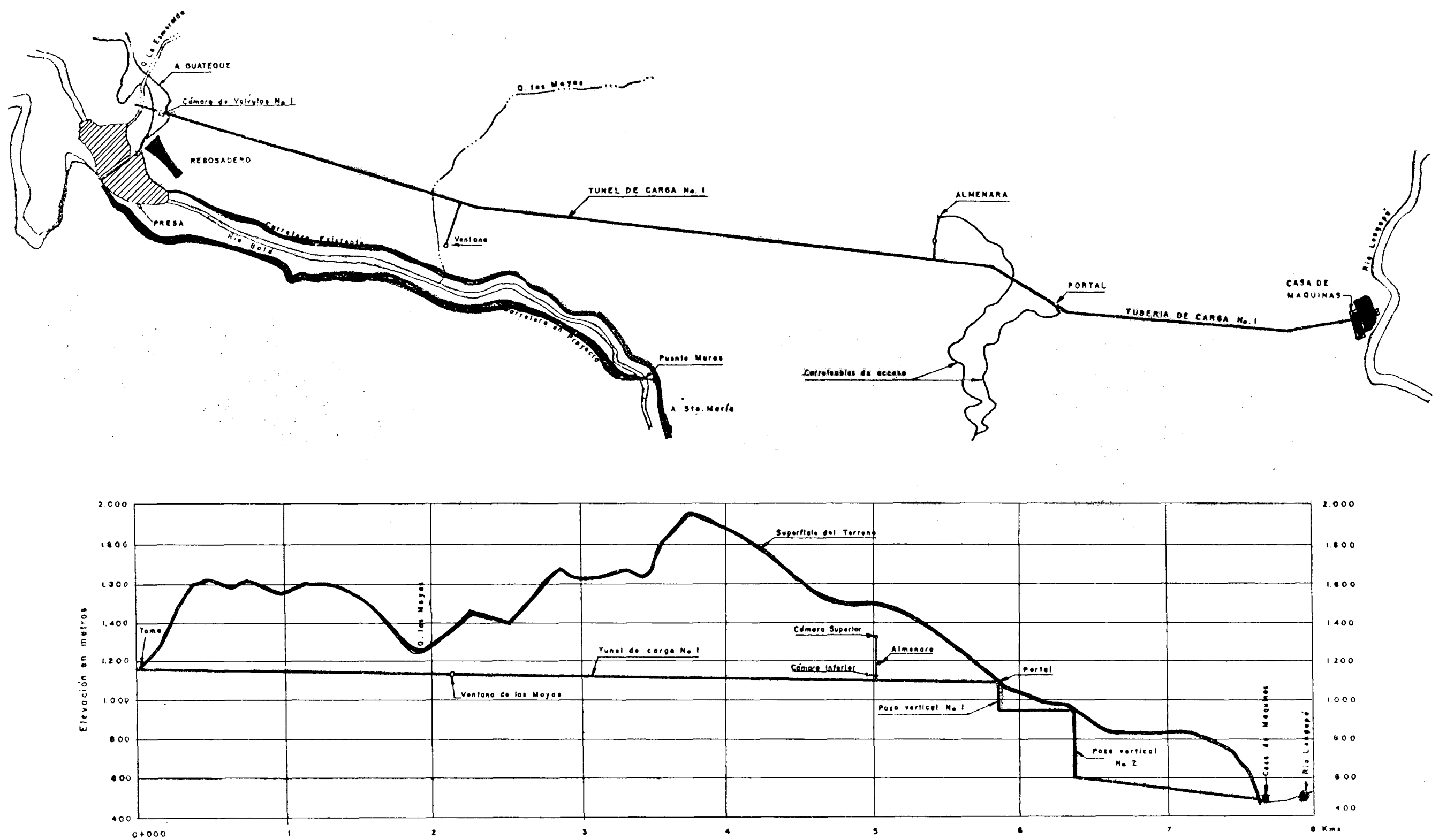

perfil conducción etapa 1 

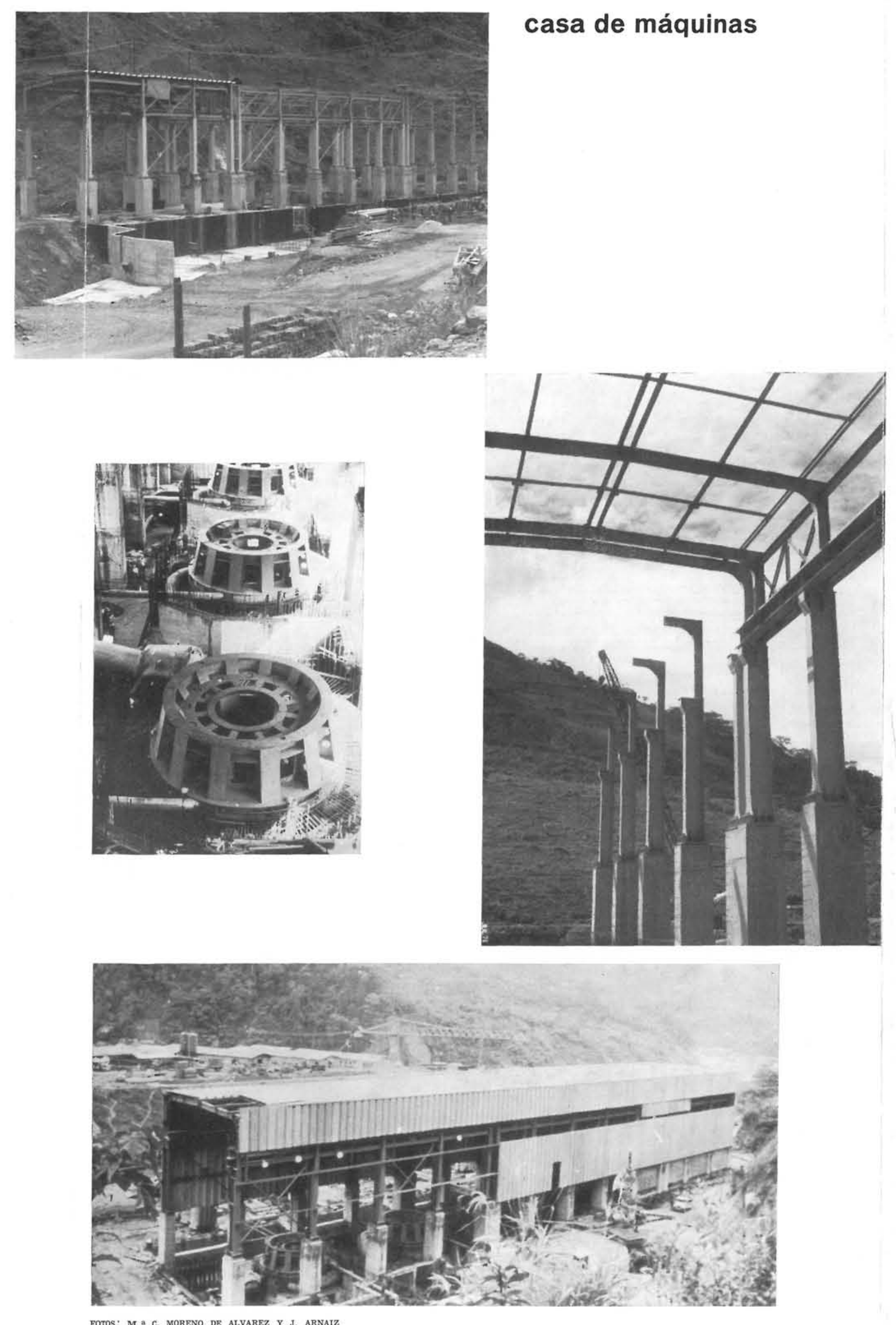

\section{Embalse}

La presa de La Esmeralda, al detener el curso de las aguas del río Batá, almacenará a lo largo de $22 \mathrm{~km}$ un total de 815 millones de $\mathrm{m}^{3}$ de agua, inundando así un área de 1.260 hectáreas, incluyendo parte de la antigua carretera que conducía a Santa María bordeando el río Batá. Por esta razón fue necesario construir una nueva carretera, de $21 \mathrm{~km}$ de longitud, en la cual existen 13 túneles con una longitud total de $6.100 \mathrm{~m}$ y puentes metálicos de longitudes diferentes, como son: Juntas $(221 \mathrm{~m})$, Pozo Azul $(115 \mathrm{~m})$, Quebradahonda $(46 \mathrm{~m})$ y el Datil $(34 \mathrm{~m})$.

\section{Rebosadero}

Sobre el estribo izquierdo de la presa se construyó un rebosadero con capacidad para evacuar $10.600 \mathrm{~m}^{3} / \mathrm{s}$, regulables con tres compuertas radiales. Sobre las pilas de hormigón se construyó un puente que comunica la carretera con la corona de la presa. Las compuertas se operan mediante controles automáticos.

La rampa del rebosadero consiste en una sección rectangular con $5 \%$ de pendiente en los primeros $122 \mathrm{~m}$, una curva parabolica de $80 \mathrm{~m}$ de longitud y $62 \mathrm{~m}$ de una parte final inclinada en un $57,7 \%$ que termina con un deflector.

\section{Túnel de carga}

El agua que alimenta las turbinas de la casa de máquinas, en su primera etapa del proyecto, es conducida hasta alli por medio de un túnel de carga de $5.830 \mathrm{~m}$ de longitud, construido mediante la excavación de $170.000 \mathrm{~m}^{3}$ de roca. Para acelerar el ritmo de trabajo durante la construcción, ésta se acometió por varios frentes; con este fin se construyo una ventana de $350 \mathrm{~m}$ de longitud. Sus primeros $5.300 \mathrm{~m}$ a partir de las tomas tien una sección en forma de herradura de $5,4 \mathrm{~m}$ de diámetro y un recubrimiento de $0,30 \mathrm{~m}$; los restantes $530 \mathrm{~m}$ tienen una sección circular con blindaje de acero y un diámetro interior de $4 \mathrm{~m}$.

El caudal del diseño es de $83 \mathrm{~m}^{3} / \mathrm{s}$ y una velocidad de $3,6 \mathrm{~m} / \mathrm{s}$.

\section{Tubería de carga}

La tubería de carga comprende dos pozos verticales de 182 y $267 \mathrm{~m}$ de altura y dos túneles que con una pendiente del $8 \%$ suman en su totalidad $2.100 \mathrm{~m}$.

\section{Bocatomas}

Las estructuras de toma para la primera y segunda etapa del proyecto fueron construidas durante la primera etapa. Consisten en dos pozos verticales de $10 \mathrm{~m}$ de profundidad, en hormigón, provistos de una estructura rectangular de rejas de $10 \mathrm{~m}$ de altura. 
Sus $237 \mathrm{~m}$ de altura la convierten en la tercera más alta en su tipo, sólo sobrepasada por en el Canadá, con $242 \mathrm{~m}$; además ocupa el séptimo lugar entre las presas más altas del mundo.
Esta presa, cuya base tiene una anchura de $820 \mathrm{~m}$, sobre el lecho del río, llega a tener una longitud de $280 \mathrm{~m}$ en cresta, lo cual permite el tráfico rodado y sirve de comunicación entre la carretera Muros-Somondoco y el puente sobre el rebosadero.

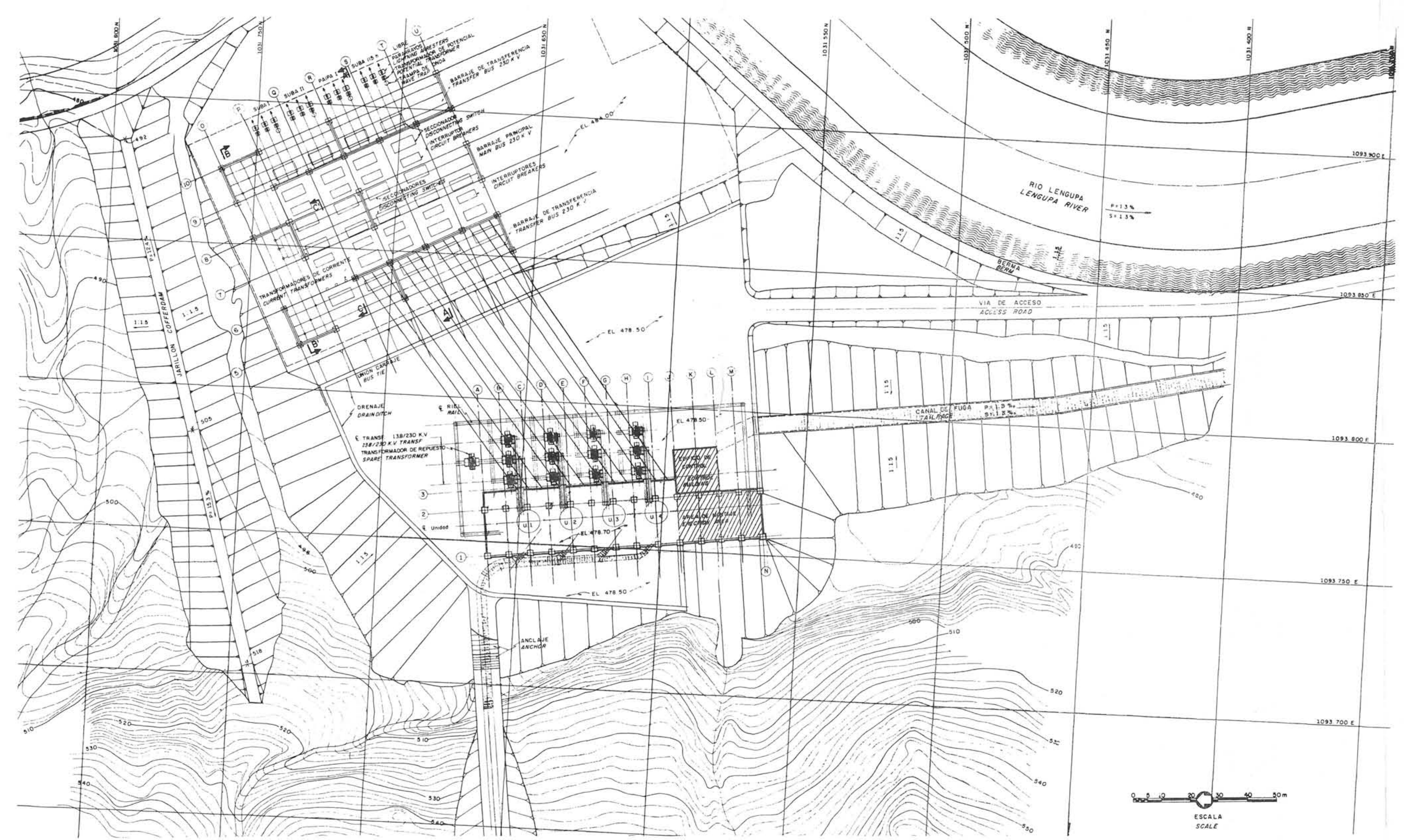

planta general 
A unos $130 \mathrm{~m}$ de esta estructura, en el túnel de carga se ha colocado una válvula mariposa de $4 \mathrm{~m}$ de diámetro, de construcción muy robusta, y a la cual se llega por una galería construida para este fin.

\section{Casa de máquinas}

Tanto la subestructura como la estructura media de casa de máquinas es de hormigón reforzado, mientras la superestructura está construida con pórticos metálicos, cubierta con placas de asbesto-cemento. Las dos etapas de casa de máquinas están separadas por el área de montaje que se ha construido en la primera etapa y presta servicio a las ocho unidades. En frente de esta zona se construyó un edificio donde funcionan los controles y las comunicaciones. Se han separado los patios de transformadores de las dos etapas del proyecto. Esta casa, que tendrá una longitud total de $180 \mathrm{~m}$ y una anchura de 16, estará provista de dos puentes-grúa de 120 t de capacidad, que corren a lo largo del eje longitudinal de la casa para la instalación y mantenimiento del equipo.

El sistema de generación de cada etapa del proyecto consiste en cuatro turbinas tipo Pelton de eje vertical, acopladas directamente al eje del generador. Cada turbina, que tiene seis chorros y puede producir $175.000 \mathrm{HP}$ a 450 r.p.m., va provista de reguladores electrohidráulicos para el control de velocidad y permite el manejo de todas como si fuera una sola, o bien el control independiente de cada una. Los generadores, con una capacidad de $140 \mathrm{MVA}$, están directamente conectados a transformadores, de 13,8 a $230 \mathrm{kV}$, los cuales, a su vez, van conectados a la subestación. La central de Chivor se unirá al sistema de interconexión mediante la línea de doble circuito Chivor-Torca-La Mesa, de $230 \mathrm{~kW}$, y con una longitud de $160 \mathrm{~km}$. Posteriormente se construirá otra línea a $230 \mathrm{~kW}$ con una longitud de $110 \mathrm{~km}$ para atender la zona nordeste del sistema interconectado; ésta será la línea Chivor-Paipa.

\section{résumé}

Hydro-électrique de Chivor. Colombile

Gabriel Tobar Guzmán, AICEMCO

Avec le projet de Chivor commença la construction de la centrale hydro-électrique la plus grande de la Colombie. Cette centrale, située à $160 \mathrm{~km}$ au nord-ouest de Bogotá, sur la Bata, au Département de Boyaca, près de Santa María, aura une capacité de $1.000 .000 \mathrm{~kW}$ et sera développée en deux étapes de $500.000 \mathrm{~kW}$ de capacité chacune. La première unité de $125.000 \mathrm{~kW}$ fut mise en service vers le milieu de 1976 et la étape de $500.000 \mathrm{wW}$, était prête en décembre 1976. La mise en service des unités 5 à 8 est prévue pour 1978.

Dans cet article sont décrits le barrage La Esmeralda, d'enrochement avec noyau imperméable d'argile et $237 \mathrm{~m}$ de hauteur, considéré comme étant le troisième le plus haut du monde dans ce genre de barrages; la retenue; le déversoir; le tunnel de charge; la conduite

\section{summary}

Chivor hydroelectric power plant. Colombia

Gabriel Tobar Guzmán, AICEMCO

The Chivor hydroelectric power plant is the largest ever built in Colombia. This plant, which is located $160 \mathrm{~km}$ to the northwest of Bogota, on the Bata River, in the Bocaya Province near Santa Maria, will have a capacity of $1.000 .000 \mathrm{~kW}$ and its development will take place in two stages of $500.000 \mathrm{~kW}$ capacity each. The first 125.000 $\mathrm{kW}$ unit was commissioned in the middle of 1976 and the fourth unit to complete the first $500.000 \mathrm{~kW}$ stage was ready in Decemthrough 8 are programmed for commissioning in 1978 .

This article describes the Esmeralda dam, rock, with an impermeable clay core, $237 \mathrm{~m}$ high, considered as the third tallest in the world, of this type; the reservoir, the overflow; the loading tunnel; the loading pipe network; and the machinery

\section{zusammenfassung}

Hydroelektrische station von Chivor. Kolumbien

Gabriel Tobar Guzmán, AICEMCO

Mit dem Projekt von Chivor begann die Konstruktion des grössten Wasserkraftwerkes von Kolumbien. Dieses Kraftwerk, das sich im Nordwesten und 160 Kilometer entfernt von Bogotá, über dem Fluss Batá, im Bezirk Boyacá, in der Nähe von Santa María, befindet wird eine Kapazität von 1.000.000 kW haben. Seine Verwirklichung wird in zwei Etappen von je $500.000 \mathrm{~kW}$ Kapazität durchgeführt. Die erste Einheit mit $125.000 \mathrm{~kW}$ wurde Mitte 1976 in $\mathrm{Be}$ trieb genommen und die vierte Einheit, die die erste Etappe von $500.000 \mathrm{~kW}$ vervollständigt, wurde im Dezember 1976 fertiggestellt. Die Inbetriebnahme der Einheiten 5 bis 8 ist für 1978 geplant.

In diesem Artikel werden beschrieben: Talsperre La Esmeralda, aus Bruchsteinschüttung mit wasserdichtem Tonkern, und 237 Meter Höhe. Man zählt sie zu den dreí Höchsten der Welt in dieser Art; das Staunel; Beschickungsleitungen und das Ma. chinenhaus. 